\title{
AN AUTOMATED 3D INDOOR TOPOLOGICAL NAVIGATION NETWORK MODELLING
}

\author{
Ali Jamali ${ }^{\mathrm{a}}$, Alias Abdul Rahman ${ }^{\mathrm{a}}$, Pawel Boguslawski ${ }^{\mathrm{b}}$ and Christopher M. Gold ${ }^{\mathrm{a}}$ \\ aUniversiti Teknologi Malaysia (UTM) \\ Faculty of Geoinformation and Real Estate \\ ali.jamali.65@gmail.com, alias@utm.my \\ chris.gold@gmail.com \\ ${ }^{b}$ University of the West of England \\ Faculty of Environment and Technology \\ Pawel.boguslawski@uwe.ac.uk
}

KEY WORDS: Indoor Surveying, Automation, 3D Data Modelling, Indoor Navigation, Topology

\begin{abstract}
Indoor navigation is important for various applications such as disaster management and safety analysis. In the last decade, indoor environment has been a focus of wide research; that includes developing techniques for acquiring indoor data (e.g. Terrestrial laser scanning), 3D indoor modelling and 3D indoor navigation models. In this paper, an automated 3D topological indoor network generated from inaccurate 3D building models is proposed. In a normal scenario, 3D indoor navigation network derivation needs accurate 3D models with no errors (e.g. gap, intersect) and two cells (e.g. rooms, corridors) should touch each other to build their connections. The presented 3D modeling of indoor navigation network is based on surveying control points and it is less dependent on the 3D geometrical building model. For reducing time and cost of indoor building data acquisition process, Trimble LaserAce 1000 as surveying instrument is used. The modelling results were validated against an accurate geometry of indoor building environment which was acquired using Trimble M3 total station.
\end{abstract}

\section{INTRODUCTION}

People spend almost 90 percent of their life in indoor building environment (Klepeis et al. 2001; Li and Lee 2010). Indoor building navigation is therefore necessary for moving objects like human to navigate. Indoor building navigation model has different challenging issues such as suitability of 3D building models, indoor navigation networks, vertical and horizontal connectivity, which require to be addressed.

Different methods are used for indoor building navigation (Zlatanova and Baharin 2008; Stoffel et al. 2007; Lamarche and Donikian 2004; $\mathrm{Li}$ and $\mathrm{He}$ 2008; Li et al. 2010; Gilliéron and Merminod 2003; Lee 2004; Yuan and Schneider 2010; Goetz and Zipf 2011) which mostly are based on the 2D floor plan or simple 3D models of buildings. Geometric Network Model (GNM) is widely proposed as suitable navigable network (Gröger and Plümer 2010; Choi and Lee 2009). A GNM is a graph consisting of nodes and edges in which nodes represent position or location of an object such as a room and edges represents connection between nodes. Li and Lee (2010) attempted to integrate GNM with Indoor GML. Luo et al. (2014) proposed generation of GNM from 3D imaging and scanning technologies.
In this paper, an automated 3D modeling of topological indoor navigation network is presented. In this approach, surveyed benchmarks are considered as dual node and generated 3D building model is considered as primal graph. Indoor navigation network is modeled using surveying benchmarks which are connected based on Delaunay triangulation.

This paper is organized as follows: Section 2 presents background of indoor building navigation. Section 3 elaborates literature review of indoor building surveying. Section 4 presents current study of indoor topological navigation network. Result of proposed 3D indoor topological navigation network is evaluated in section 5 . Conclusion and future research is discussed in section 6 .

\section{BACKGROUND}

According to Liu and Zlatanova (2011), an up to date and accurate navigation model to be considered is most crucial requirement for developing a robust emergency response system: semantically rich knowledge, automated generation of network, dynamic routing capacity and door-to-door movement capability are some of the requirements in indoor navigation modelling. 
Semantically rich knowledge provides useful information about geometrical object such as door locations or exit points in a building. As shown in Figure 1, Liu and Zlatanova (2012) explained door to door route as "the straight medial axis of a corridor is D1-M1-M2-M3-M4-D4. Even if the person could not see the door D3 from D1, the "door to door" route could be D1-S1-D3 (the dash line), which means the person will see $D 3$ until he reaches $S 1$. Yet the dual graph route would be D1-M1-M2-M3-D3 in this case”.

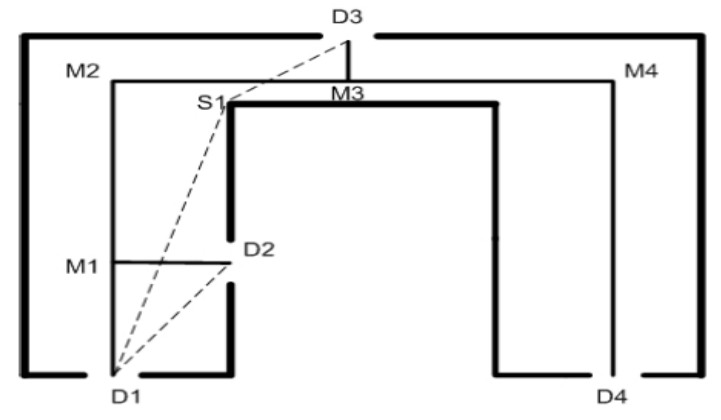

Figure 1. An example of door to door routes (Liu and Zlatanova, 2011)

Existing indoor navigation models as mentioned by Liu and Zlatanova (2011) are as follows:

Lee (2001) proposed conventional dual based on the Poincare Duality theory. For connectivity of building, conventional dual uses Node-Relation Structure (NRS). GNM was an extension of NRS to represent more accurate indoor building environment (Lee, 2004). For improving construction of GNM, a skeleton-abstraction algorithm was proposed by Lee (2004), which is based on the StraightMedial Axis Transformation (S-MAT). Becker et al. (2009) proposed a Multilayered Space-event which in their model each space layer is divided into primal and dual space with topology and geometry division of spaces.

Many researchers have used Conventional Dual Graph (Lee 2004; Meijers et al. 2005; Li and He 2008; Becker et al. 2009; Boguslawski et al. 2011). To improve S-MAT, Yuan and Schneider (2010) proposed Direct Path Graph (DPG) which consists of cells, path segments and accessibilities.

Navigable space model (Slingsby and raper 2008, Schaap 2010) is topologically-connected and navigable space (surfaces) which leads to pedestrian's movement. Navigable space model is a $2.5 \mathrm{D}$ reconstructed model of 2D plan with limited height and space constraints. Network generation in complex scenarios and automatic generation of network are challenging and there is no door-to-door movement in this model.

Yuan and Schneider (2010) proposed LEGO-graph which is a Regular-grid graph based on the 3D voxel; LEGOgraph using different width and heights can provide all possible paths. The Regular-grid model can be a $2 \mathrm{D}$ or $3 \mathrm{D}$ model. 3D Regular-grid model is an extension of 2D model. 3D model voxel could be used to represent indoor building structure. In Regular-grid graph nodes represent grid and edges of a specific node represent relationship between the node and its surrounding neighbors ( $\mathrm{Li}$ et al. 2009). Bandi and Thalmann (1998) discredited building parts into 3D voxel to compute an obstacle-free route with surmountable and insurmountable obstacle.

Considering several criteria (e.g. distance between walls, visibility) the Sub-division intends to divide 2D plans into cells. Lamarche and Donikian (2004) used constrained Delaunay triangulation algorithm and Convex Cell optimization to subdivide a 2D plan. Lorenz et al. (2006) divided a 2D plan into cells to create a graph structure. As shown by Figure 2, in their model Cell centers are connected to doors.

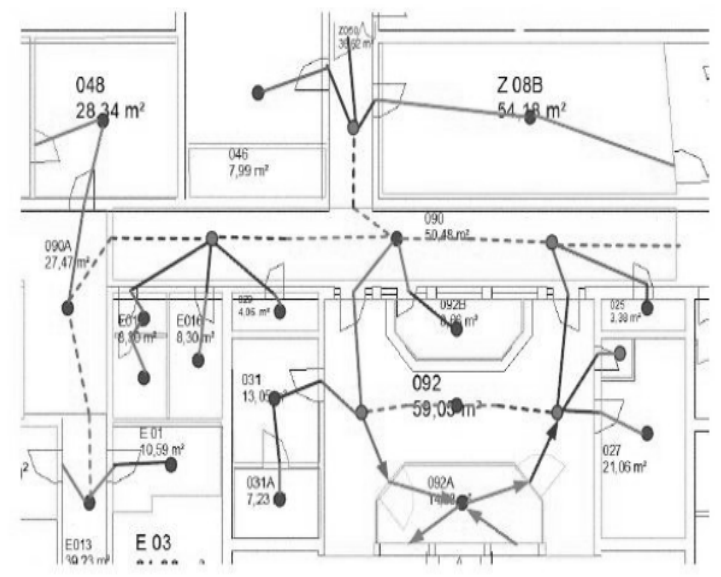

Figure 2. Cell centers and paths overlaid with a floor Plan (Lorenz et al. 2006)

Stoffel et al. (2007) used visibility criteria to divide a plan into cells. As can be seen in Figure 3, in their model, a plan is partitioned into non-overlapping convex sub-regions (cells) according to visibility conditions.

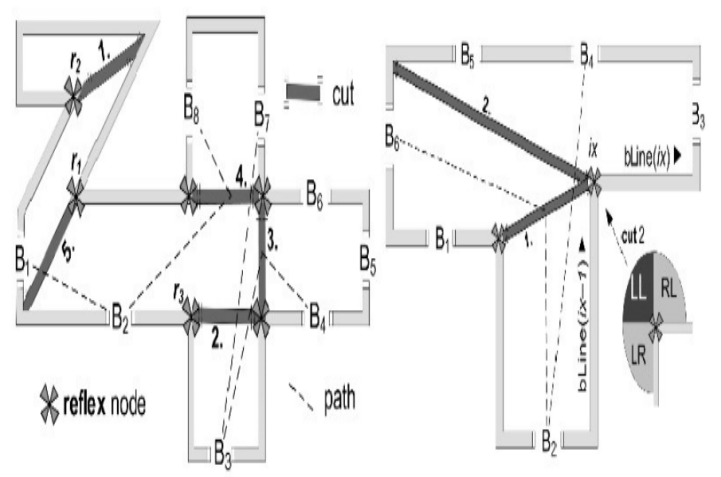

Figure 3. Visibility partitioning result (Stoffel et al. 2007) 
Liu and Zlatanova (2011) proposed Indoor Visibility Structure (IVS) and According to Liu and Zlatanova (2011) a $3 \mathrm{D}$ sub-division with visibility consideration is more accurate and suitable approach for indoor navigation routing as shown by Figure 4. "Possible outlets encompass inner room doors (denoted by "I"), room doors (denoted by "D"), stairs (denoted by "S") and windows (denoted by "W"). Each opening is abstracted as a node of a network model (see in the bottom-left part of Figure 4). If two openings are mutually visible to each other, then there is an edge of the network between the two nodes. The dash-line edges in Figure 4 indicate windows will be used for escape only in some specific conditions (e.g. broken in an emergency situation)”.

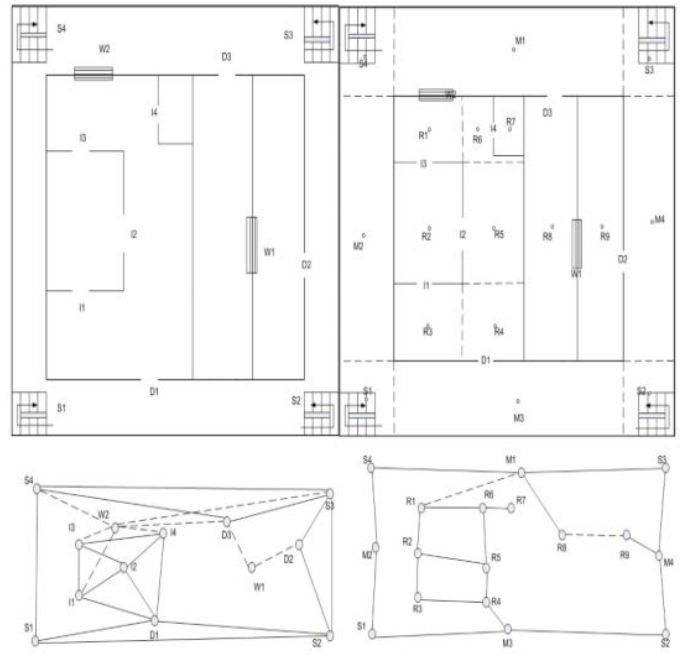

Figure 4. Indoor visibility structure and conventional dual graph model (Liu and Zlatanova, 2011)

\section{DATA COLLECTION}

For reducing time and cost of indoor building data acquisition process, Trimble LaserAce 1000 as surveying instrument is used. The Trimble LaserAce 1000 has been used for outdoor mapping and measurements, such as forestry measurement and GIS mapping. A rangefinder can be considered as a basic mobile Total Station with limited functionality and low accuracy as shown in Figure 5 . Trimble LaserAce 1000 will decrease time and cost of surveying process (Jamali et al. 2013; Jamali et al. 2014).

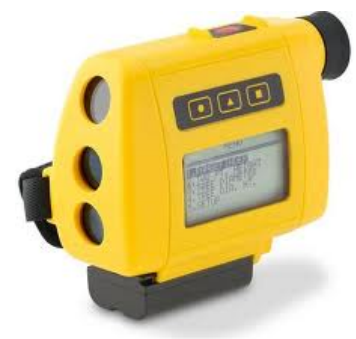

Figure 5. Surveying device: Trimble LaserAce 1000
According to the device specifications, the accuracy of the Trimble LaserAce 1000 is as shown in Table 1.

Table 1. Accuracy of Trimble LaserAce 1000 according to the product specifications.

\begin{tabular}{llll}
\hline $\begin{array}{l}\text { Surveying } \\
\text { Equipment }\end{array}$ & $\begin{array}{l}\text { Distance } \\
\text { Accuracy }\end{array}$ & $\begin{array}{l}\text { Horizontal } \\
\text { Angle } \\
\text { Accuracy }\end{array}$ & $\begin{array}{l}\text { Vertical } \\
\text { Angle } \\
\text { Accuracy }\end{array}$ \\
\hline $\begin{array}{l}\text { Trimble } \\
\text { LaserAce } \\
1000\end{array}$ & $\pm 100 \mathrm{~mm}$ & $7200 ”$ & $720 ”$ \\
& & & \\
\hline
\end{tabular}

The geometry of rooms and corridors is not required to be accurate in this particular work due to the topological reconstruction of the network (see Figure 6). The presented 3D modeling of indoor navigation network is based on surveying control points. Geometry of rooms and corridors are considered as one of the factors for the connection between neighboring rooms (in the proposed method, to connect two neighboring rooms; geometry of rooms and corridor will be checked to insure their neighborhood). In order to establish surveying benchmarks as control points, closed traverse surveying was used which is a method of surveying for establishing control points along traveling or movement path. Closed traverse surveying will start from one point with known coordinates and will end in the same point.

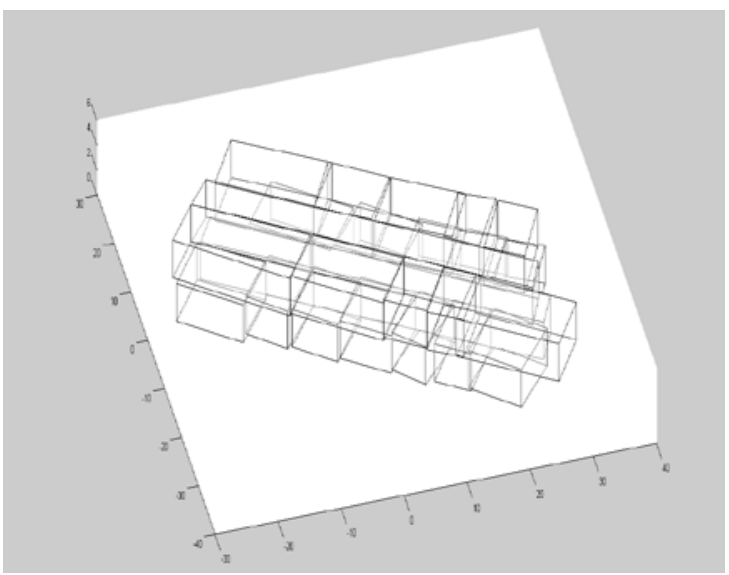

Figure 6. 3D building modelling (using MATLAB computing language) by Trimble LaserAce 1000

\section{PROPOSED INDOOR NAVIGATION NETWORK}

In the proposed indoor navigation network modeling, surveying benchmarks are considered as dual node while the generated 3D model is represented as a primal graph. Each room, corridor and benchmark has a specific ID which is referred as a primary key. Surveying benchmarks and building model are connected in accordance with their IDs, as shown in Figure 7. 


\begin{tabular}{|c|c|}
\hline Dual Node (Survoying Benchmarks) & Primal Graph(3D Building Models) \\
\hline $\begin{array}{l}\text { Benchmark ID } \\
\text { Room ID OR Corridor ID }\end{array}$ & -Room ID OR Corridor ID \\
\hline
\end{tabular}

Figure 7. Semantic information associated to dual and primal graph

Semantic information is used for the recognition of two neighboring rooms or corridors. Each Primal graph contains one or several dual nodes which can be utilized in the further processes (ID of rooms and corridors will be used for the topological reconstruction).

The indoor navigation network modeling consists of two main procedures - $3 \mathrm{D}$ modelling and navigation networking. These procedures can be further explained in five steps as follows:

1. In the indoor navigation modeling, rooms and corridor coordinates are input as first step.

Geometry of each room and corridor constitutes at least eight vertices along with their $\mathrm{x}, \mathrm{y}$ and $\mathrm{z}$ coordinates. These coordinate vertices for each room and corridor will be read in this step as seen in Figure 8.

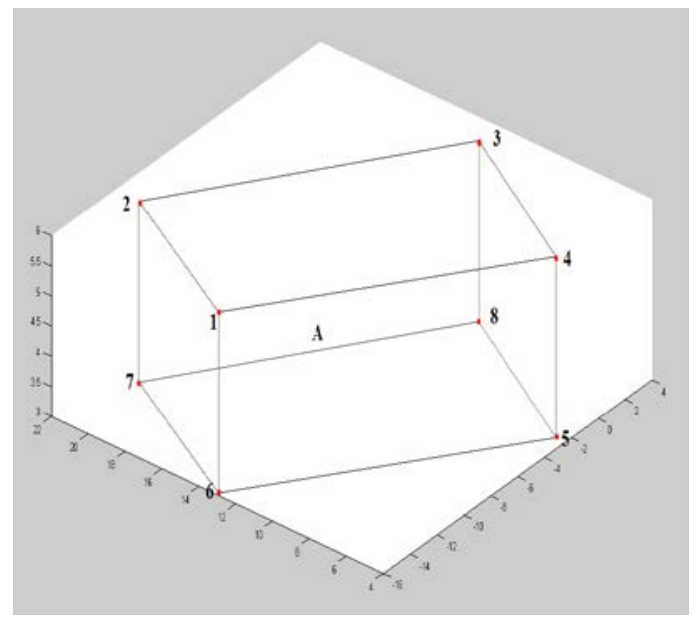

Figure 8. A room block where vertices are represented as red points

2. In the second step, surveying benchmarks are input as control points. As shown in Figure 9, $x$, $\mathrm{y}$ and $\mathrm{z}$ coordinates of surveying benchmarks coordinates are read. These surveying control points belonging to doors, elevators, stairs, rooms and corridor are considered as dual nodes.

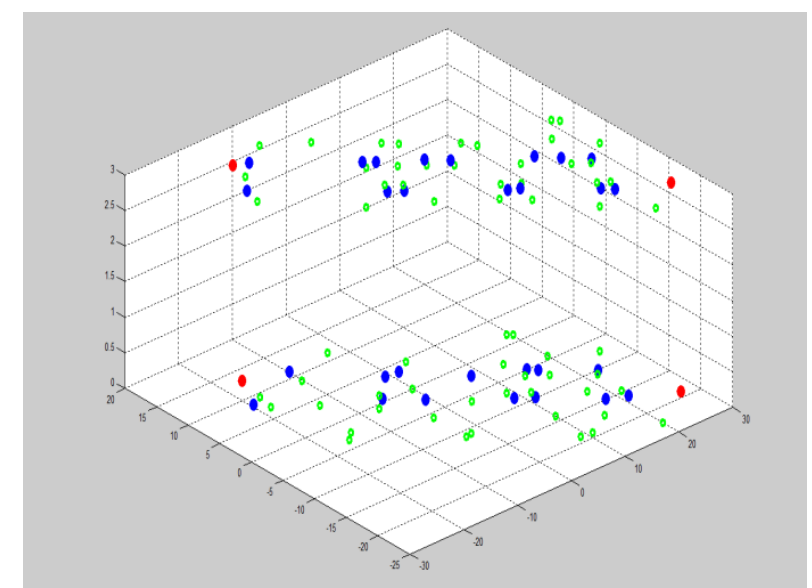

Figure 9. Surveying control points as dual nodes: doors are represented as blue points, elevators as red points while room and corridor control points as green points.

3. In the third step, 3D modeling of indoor building environment is done based on input 3D coordinates of rooms and corridor (see Figure 6).

In this research, 3D modelling is based on boundary representation with using vertices and faces. Due to low accuracy of Trimble LaseAce 1000 rangefinder, 3D geometrical building model is not as much accurate as there are gaps and intersections between rooms and corridors as seen in Figure 6.

4. In the fourth step, connection between control points is generated.

Topological connection between control points is generated using Delaunay triangulation. The connection of control points using Delaunay triangulation facilities navigation and neighborhood queries along rooms and corridor network. There are three conditions for connecting control points. If there is one control point, then there will be no connection. If there are two control points, then there will be one connection between the control points. In case of three or more control points, connection is made using Delaunay triangulation method as shown in Figure 10. 


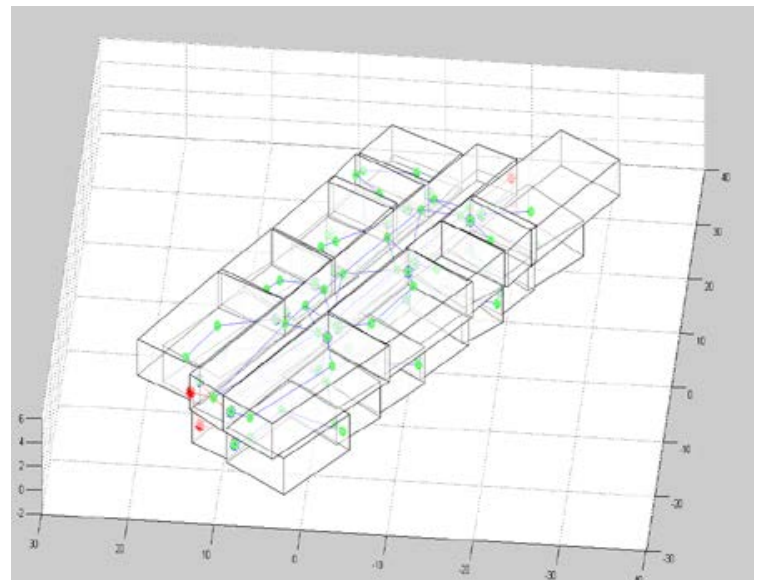

Figure 10. Connection between three or more control points (green points) using Delaunay triangulation (blue lines).

5. In the fifth step, connection between rooms is generated.

In order to build connection, three different scenarios i.e. gap, intersect and touch are considered. Connection between rooms (see Figure 11) has been considered as it is important for various applications such as disaster management and safety analysis, for example, if there is a need to break walls between two rooms. Due to low accuracy of laser rangefinder, the modeled shape of building might be inaccurate, therefore, two neighboring rooms might intersect each other, there might be a gap between them and in the best scenario they might touch each other.

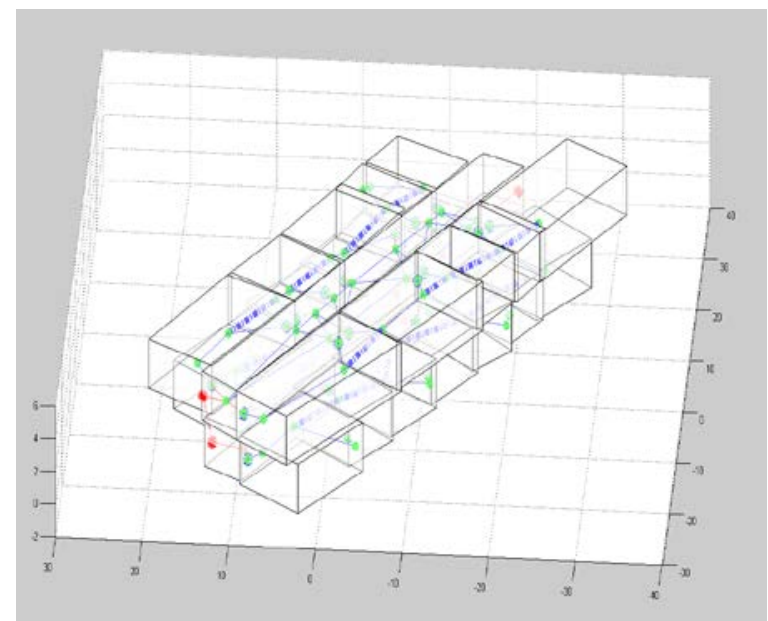

Figure 11. Proposed 3D indoor topological navigation network where surveying control points are represented as green points, connection between dual nodes as blue lines, connection between rooms as blue dash lines and connection between floors as red lines.

\section{VALIDATION OF TOPOLOGICAL NETWORK AND PERFORMANCE ANALYSIS}

The indoor navigation network result was validated with the network model generated using an accurate dataset. This accurate data of indoor building environment was acquired using Trimble M3 total station which led to the generation of 3D building model without any gap between cells (i.e. rooms and corridor) as seen in Figure 12.

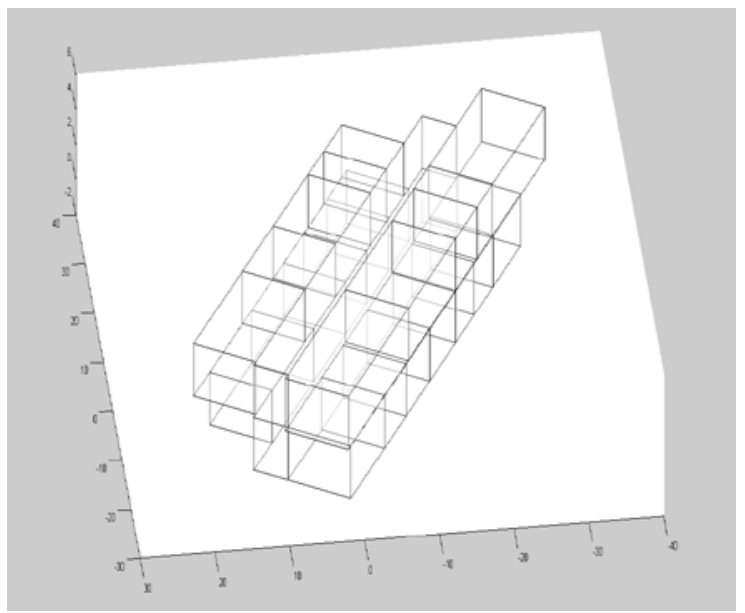

Figure 12. 3D building modelling using Trimble M3 total station.

The topological indoor navigation network models generated using Trimble LaserAce 1000 rangefinder and Trimble M3 total station are shown in Figure 13.

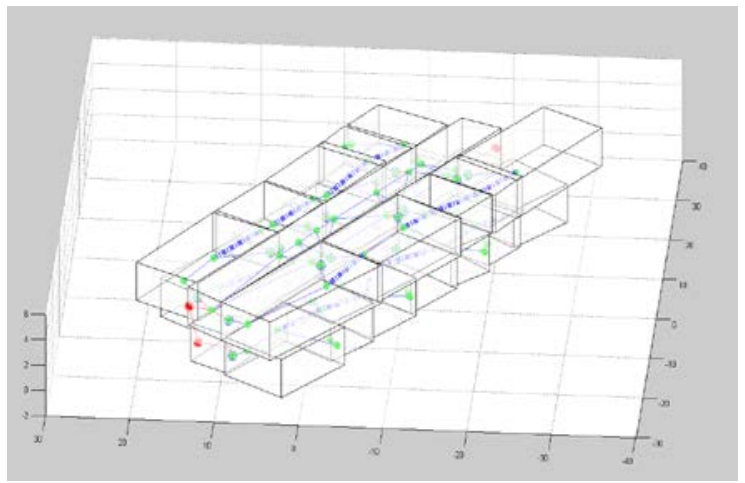

(a) 


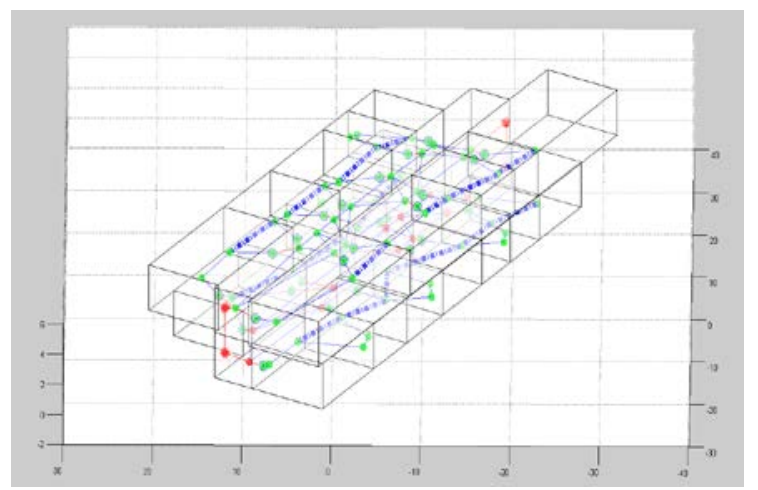

(b)

Figure 13. Topological indoor navigation network model generated using (a) Trimble LaserAce 1000 rangefinder and (b) Trimble M3 total station.

It can be proposed that the topological indoor navigation network is less dependent on the $3 \mathrm{D}$ geometrical building model. The accurate 3D geometrical building model is only required for establishing connection between rooms and neighborhood queries.

The proposed indoor topological navigation network is fully automated. This 3D modelling was performed on a computer withi7 core@3.4 GHz processor, 8 GB RAM and 64-bit operating system. For the development of the proposed 3D modelling, MATLAB computing language is used (see Figure 14).

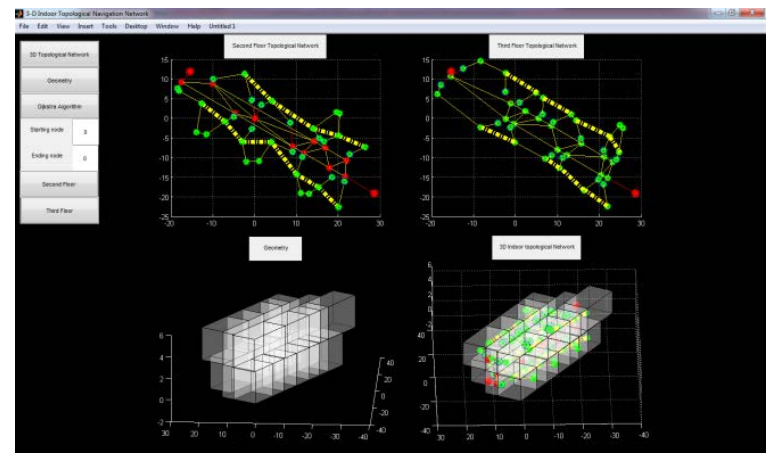

Figure 14. Developed Graphical User Interface using MATLAB computing language.

\section{CONCLUSION}

In this paper, an automated 3D modeling of indoor navigation network has been presented. Trimble LaserAce 1000 was used to acquire data of indoor building environment. Results from such surveying equipment are inaccurate. The indoor navigation network model includes two main procedures including 3D building modelling and topological navigation networking. Connection between controls points is established based on the Delaunay triangulation.
The presented topological modelling uses semantic and geometrical information and does not require an accurate geometric model. It can be proposed that the navigation network can also be generated using less accurate and cheap surveying instrument (i.e. Trimble LaserAce 1000 rangefinder) which decreases time and cost for acquiring data in indoor building environment.

Nowadays, municipalities intend to have 3D city models for facility management, disaster management and architectural planning. 3D data acquisition can be done by laser scanning for indoor environment which is costly and time consuming process. We believe that the proposed surveying technique can be employed for basic indoor environment modelling to decrease cost and time for generating 3D city models. The proposed surveying technique could be utilized for most of municipalities. Future research attempts to investigate performance and efficiency of the proposed indoor topological navigation network against an accurate geometry of indoor building environment.

\section{REFERENCES}

Amato, L., Antonucci, G., \& Belnato, B. (2003). The three dimensional laser scanner system: The new frontier for surveying. Case history: The leaning tower of PISA (ITALY), The ancient theatre of Taormina (Italy), The prehistoric site of Nola (Naples-Italy). International Archives of Photogrammetry Remote Sensing and Spatial Information Sciences, 34(5/w12), 17-22.

Bandi, S., \& Thalmann, D. (1998, August). Space discretization for efficient human navigation. In Computer Graphics Forum (Vol. 17, No. 3, pp. 195-206). Blackwell Publishers Ltd.

Becker, T., Nagel, C., \& Kolbe, T. H. (2009). A multilayered space-event model for navigation in indoor spaces. In 3D Geo-Information Sciences (pp. 61-77). Springer Berlin Heidelberg.

Boguslawski, P., Gold, C. M., \& Ledoux, H. (2011). Modelling and analysing 3D buildings with a primal/dual data structure. ISPRS Journal of Photogrammetry and Remote Sensing, 66(2), 188-197.

Dongzhen, J., Khoon, T. Y., Zheng, Z., \& Qi, Z. (2009). Indoor 3D Modeling and Visualization with a 3D Terrestrial Laser Scanner. In 3D Geo-Information Sciences (pp. 247-255). Springer Berlin Heidelberg.

Gröger, G., \& Plümer, L. (2010). Derivation of 3D indoor models by grammars for route planning. PhotogrammetrieFernerkundung-Geoinformation, 2010(3), 191-206.

Goetz, M., \& Zipf, A. (2011). Formal definition of a useradaptive and length-optimal routing graph for complex indoor environments. Geo-Spatial Information Science, 14(2), 119-128. 
Gilliéron, P. Y., \& Merminod, B. (2003, October). Personal navigation system for indoor applications. In 11th IAIN world congress (pp. 21-24).

Jamali, A., Boguslawski, P., Duncan, E. E., Gold, C. M., \& Rahman, A. A. (2013). Rapid Indoor Data Acquisition for Ladm-Based 3d Cadastre Model. ISPRS Annals of Photogrammetry, Remote Sensing and Spatial Information Sciences, 1(1), 153-156.

Jamali, A., Boguslawski, P., Gold, C. M., \& Rahman, A. A. (2014). Rapid Indoor Data Acquisition Technique for Indoor Building Surveying for Cadastre Application. In Innovations in 3D Geo-Information Sciences (pp. 1-11). Springer International Publishing.

Klepeis, N. E., Nelson, W. C., Ott, W. R., Robinson, J. P., Tsang, A. M., Switzer, P., ... \& Engelmann, W. H. (2001). The National Human Activity Pattern Survey (NHAPS): a resource for assessing exposure to environmental pollutants. Journal of exposure analysis and environmental epidemiology, 11(3), 231-252.

Lamarche, F., \& Donikian, S. (2004, September). Crowd of virtual humans: a new approach for real time navigation in complex and structured environments. In Computer Graphics Forum (Vol. 23, No. 3, pp. 509-518). Blackwell Publishing, Inc.

Lee, J. (2001). 3D data model for representing topological relations of urban features. In Proceedings of the 21st Annual ESRI International User Conference, San Diego, CA, USA.

Lee, J. (2004). A spatial access-oriented implementation of a 3-D GIS topological data model for urban entities. GeoInformatica, 8(3), 237-264.

Li, Y., \& He, Z. (2008). 3D indoor navigation: a framework of combining BIM with 3D GIS. In 44th ISOCARP congress.

Liu, L., \& Zlatanova, S. (2011, May). A" door-to-door" Path-finding Approach for Indoor Navigation. In Proceedings Gi4DM 2011: GeoInformation for Disaster Management, Antalya, Turkey, 3-8 May 2011. International Society for Photogrammetry and Remote Sensing (ISPRS).

Liu, L., \& Zlatanova, S. (2011). Towards a 3D network model for indoor navigation. Urban and Regional Data Management, UDMS Annual, 79-92.

Liu, L., \& Zlatanova, S. (2013). A Two-level Path-finding Strategy for Indoor Navigation. In Intelligent Systems for Crisis Management (pp. 31-42). Springer Berlin Heidelberg.

Lorenz, B., Ohlbach, H. J., \& Stoffel, E. P. (2006). A hybrid spatial model for representing indoor environments. In Web and Wireless Geographical Information Systems (pp. 102-112). Springer Berlin Heidelberg.
Luo, F., Cao, G., \& Li, X. (2014, November). An interactive approach for deriving geometric network models in 3D indoor environments. In Proceedings of the Sixth ACM SIGSPATIAL International Workshop on Indoor Spatial Awareness (pp. 9-16). ACM.

Penninga, F., \& Van Oosterom, P. (2007). A compact topological DBMS data structure for 3D topography. In The European Information Society (pp. 455-471). Springer Berlin Heidelberg.

Schaap, J., Zlatanova, S., \& van Oosterom, P. J. M. (2011). Towards a 3D geo-data model to support pedestrian routing in multimodal public transport travel advices. Urban and Regional Data Management, UDMS Annual, 63-78.

Slingsby, A., \& Raper, J. (2008). Navigable space in 3D city models for pedestrians. In Advances in $3 D$ Geoinformation Systems (pp. 49-64). Springer Berlin Heidelberg.

Stoffel, E. P., Lorenz, B., \& Ohlbach, H. J. (2007). Towards a semantic spatial model for pedestrian indoor navigation. In Advances in Conceptual ModelingFoundations and Applications (pp. 328-337). Springer Berlin Heidelberg.

Yuan, W., \& Schneider, M. (2010, November). Supporting $3 \mathrm{D}$ route planning in indoor space based on the LEGO representation. In Proceedings of the 2nd ACM SIGSPATIAL International Workshop on Indoor Spatial Awareness (pp. 16-23). ACM.

Yuan, W., \& Schneider, M. (2010). iNav: An indoor navigation model supporting length-dependent optimal routing. In Geospatial Thinking (pp. 299-313). Springer Berlin Heidelberg.

Zlatanova, S., \& Baharin, S. S. K. (2008). Optimal navigation of first responders using DBMS. In $3 r d$ International Conference on Information Systems for Crisis Response and Management 4th International Symposium on GeoInformation for Disaster Management (pp. 541-54). 